\title{
A NEW PROOF OF THE ZERO-ONE LAW FOR STABLE MEASURES
}

\author{
W. SMOLENSKI
}

ABstract. A new proof of the zero-one law for stable measures is given.

Definition. A probability measure $\mu$ on a linear measurable space $E$ is said to be stable if $\mu^{* n}=\mu \circ T_{n}^{-1}{ }^{1}$ where $T_{n}(x)=a_{n} x+y_{n}$, for some $a_{n} \in \mathbf{R}^{1}, y_{n} \in E$; if for every $n, y_{n}=0$, then $\mu$ is said to be strictly stable.

THEOREM. Let $\mu$ be a stable probability measure on a linear measurable space $E$. Then for any linear measurable subspace $E_{1}$ of $E \mu\left(E_{1}\right)=0$ or $\mu\left(E_{1}\right)=1$.

The above theorem is due to R. M. Dudley and M. Kanter [1]. A simple but rather tricky proof was given by $X$. Fernique [2]. Here we provide another elementary proof which depends on a lemma of some independent interest.

LEMMA. Let $E$ and $E_{1}$ be as above and let $\mu$ be a probability measure on $E$. Suppose that $\mu\left(E_{1}\right)<1$. Then $\mu^{* n}\left(E_{1}\right)$ tends to zero as $n$ tends to infinity.

PROOF OF THE THEOREM. We may assume that $\mu$ is strictly stable. (Indeed, if $0<\mu\left(E_{1}\right)<1$ then the same holds for the symmetrization of $\mu$.) Suppose that $\mu\left(E_{1}\right)<1$. By the lemma $\mu^{* n}\left(E_{1}\right) \rightarrow 0$. The strict stability of $\mu$ gives $\mu^{* n}\left(E_{1}\right)=$ $\mu\left(E_{1}\right)$ and the proof is finished.

Proof of THE LEMma. Assume first that $E=\mathbf{R}^{1}$ and $E_{1}=\{0\}$. If $\mu=\delta_{a}$, then $a \neq 0$ since $\mu\left(E_{1}\right)<1$ is assumed and hence $\mu^{* n}(\{0\})=0$. If $\mu \neq \delta_{a}$, then there exists $a>0$ such that $|\hat{\mu}(x)|<1$ for $|x|<a$ where $\hat{\mu}$ is the Fourier transform of $\mu$. Letting $f_{a}(x)=a^{-1}\left(1-a^{-1}|x|\right),-a \leqslant x \leqslant a$, and zero elsewhere, we have

$$
\begin{aligned}
\int_{-\infty}^{\infty}(\hat{\mu}(x))^{n} f_{a}(x) d x & =\int_{-\infty}^{\infty}\left(\int_{-\infty}^{\infty} e^{i t x} \mu^{* n}(d t)\right) f_{a}(x) d x \\
& =\int_{-\infty}^{\infty} 2\left(\frac{1-\cos a t}{a^{2} t^{2}}\right) \mu^{* n}(d t)
\end{aligned}
$$

and hence

$$
\begin{aligned}
\mu^{* n}(\{0\}) & \leqslant \int_{-\infty}^{\infty} 2\left(\frac{1-\cos a t}{a^{2} t^{2}}\right) \mu^{* n}(d t) \\
& =\int_{-a}^{a}(\hat{\mu}(x))^{n} f_{a}(x) d x \rightarrow 0
\end{aligned}
$$

as $n \rightarrow \infty$.

Received by the editors September 29, 1980 and, in revised form, January 30, 1981.

AMS (MOS) subject classifications (1970). Primary 60B05.

Key words and phrases. Linear measurable space, stable probability measure, 0-1 law.

${ }^{1} \mu^{* n}$ denotes the $n$th power of $\mu$ in the sense of the convolution.

(C) 1981 American Mathematical Society 0002-9939/81/0000-0487/\$01.50 
Since $E_{1}$ is a linear subspace

$$
\left(\nu_{1} * \nu_{2}\right)\left(E_{1}\right)=\sum \nu_{1}\left(E_{1}-x\right) \nu_{2}\left(E_{1}+x\right),
$$

summation being taken over all cosets of $E_{1}$. (This follows directly from the convolution formula.)

If $\Sigma \mu\left(E_{1}+x\right)=a<1$, then $\mu^{* n}\left(E_{1}\right)<a^{n}$ and we are done. Hence we can assume $\Sigma \mu\left(E_{1}+x\right)=1$. In this case let $\nu$ be the image of $\mu$ on $E / E_{1}$ under the canonical quotient map; $\nu$ is purely atomic and, since $\mu\left(E_{1}\right)<1$, there exists a linear functional $f$ on $E / E_{1}$ such that $\left(\nu \circ f^{-1}\right)(\{0\})<1$. (We do not have to bother about the measurability because $\nu$ is purely atomic.) We have (by (1))

$$
\mu^{* n}\left(E_{1}\right)=\nu^{* n}(\{0\}) \leqslant\left(\nu \circ f^{-1}\right)^{* n}(\{0\}) \underset{n \rightarrow \infty}{\rightarrow} 0,
$$

which finishes the proof of the lemma.

\section{REFERENCES}

1. R. M. Dudley and M. Kanter, Zero-one laws for stable measures, Proc. Amer. Math. Soc. 45 (1974), 245-252.

2. X. Fernique, Une démonstration simple du théorème de R. M. Dudley et Marek Kanter sur les lois zéro-un pour les mésures stables, Lecture Notes in Math., vol. 381, Springer, Berlin, 1974, pp. 78-79.

Department of Mathematics, Louisiana State University, Baton Rouge, Louisiana 70803

Institute of Mathematics, Warsaw Technical University, 00-661 Warsaw, Poland (Current address) 